\title{
Gamma-ray bursts associated with supernovae: a systematic analysis of BATSE GRB candidates
}

\author{
Z. Bosnjak ${ }^{1}$, A. Celotti ${ }^{1}$, G. Ghirlanda ${ }^{2}$, M. Della Valle ${ }^{3}$, and E. Pian ${ }^{4}$ \\ 1 SISSA/ISAS, via Beirut 2-4, 34014 Trieste, Italy \\ e-mail: celotti@sissa.it \\ 2 INAF - Observatory of Brera, via Bianchi 46, 23807 Merate (LC), Italy \\ ${ }^{3}$ INAF - Observatory of Arcetri, Largo E. Fermi 5, 50125 Firenze, Italy \\ ${ }^{4}$ INAF - Observatory of Trieste, via G.B. Tiepolo 11, 34131 Trieste, Italy
}

Received 1 February 2005 / Accepted 25 September 2005

\section{ABSTRACT}

We examined the properties of a sample of BATSE Gamma-Ray Bursts (GRBs) comprising events which have indications of association with a supernova $(\mathrm{SN})$, some on the basis of indications of re-brightening in the optical afterglow light curve, but in most cases based only on the "loose" temporal and directional coincidence inferred from the cross correlation of catalogs. Despite the large uncertainties in the latter selection method, the temporal and spectral analysis reveal three interesting statistical results when the sample is compared with that of all the BATSE GRBs: the GRBs tentatively associated with SNe are found to predominantly (in $\sim 80 \%$ of the cases) have single-peaked light curves, a softer spectrum (i.e. low energy power law index $\alpha \sim-1.5$ ) and tend not to follow the Lag-Luminosity and Isotropic Energy-Peak Energy correlations. These three independent statistical properties point toward the existence of a significant number of under-luminous, GRB 980425-like events constituting - at least from an observational point of view - a tail or a separate class with respect to the whole of the BATSE GRB events. The unusually high percentage of SN Ibc among those identified by the catalog cross-correlation (a factor four higher than expected from $\mathrm{SN}$ catalog statistics) reinforces the non-randomness of some of the selected events.

Key words. gamma rays: bursts - methods: data analysis - stars: supernovae: general

\section{Introduction}

An increasing number of recent observations support the association of (at least) some GRBs with SN events, stimulating both the theoretical understanding/modeling of GRB progenitors and intensive observational campaigns aimed at finding further and more detailed evidence of associations.

GRB 980425 was the first burst with tantalizing evidence for a physical association with a SN (SN 1998bw, Galama et al. 1998). The association of the two events has been discussed in the framework of models involving the core collapse of massive stars (e.g. Woosley et al. 1999; MacFadyen \& Woosley 1999; Vietri \& Stella 1998). However, the unusual properties of both events within their classes - i.e. the high luminosity of the SN, the unusually low luminosity and variability of the GRB $\gamma$-ray emission and the lack of detected X-ray afterglow - as well as the uniqueness of this association, have not allowed investigators to draw firm conclusions about the possible progenitor.

More recently two further GRB/SN associations based on the spectroscopic detection of a SN spectrum in the GRB afterglow added robust evidence to the association of the two phenomena. GRB 030329 (Stanek et al. 2003; Hjorth et al. 2003) has been associated with SN 2003dh from the appearance of typical SN Ic spectral features in its optical spectrum and the (under-energetic) GRB 031203 revealed an optical spectrum consistent with the contribution of an underlying type Ic SN (SN 20031w; Malesani et al. 2004).

Despite these promising findings, the paucity of cases of GRB directly associated with $\mathrm{SN} \mathrm{Ib} / \mathrm{c}$, some peculiar properties of both the GRB and SN events in these few robust associations and the uncertainties on the event rates in both classes (see however Podsiadlowski et al. 2004) do not allow a clear insight and different possibilities are still open: selection effects might play a role in detecting $\mathrm{SN}$ features, there might be an (inverse) relation between the energetics of the two events (under-luminous GRB vs. Hypernova event), or bursts associated with core collapse SN could represent a subset of the whole population, which might be characterized by properties (e.g. energetics, duration, temporal variability) intermediate between the two class of events (e.g. Della Valle 2004, for an overview; Soderberg 2004).

Given the limited number of "direct" associations (i.e. with evidence of SN spectral features in the GRB afterglow), in order to test for the nature of any physical connection and thus investigate the progenitor(s) of GRB, other more indirect 
indications could be considered to select candidates GRB/SN associations. These include the re-brightening in the optical afterglow light curve decay, a possible signature of SN emission emerging at times when the GRB has sufficiently faded (Bloom et al. 1999; Germany et al. 2000). Indeed about ten such associations have been claimed on such a basis (Zeh et al. 2004). Interestingly Zeh et al. (2004) found indication of re-brightening in all GRBs with known redshift $z<0.7$. Similar evidence (i.e. a bump in the late time light curve) also have been found in the case of a few X-ray Flashes (see Fynbo et al. 2004; Soderberg et al. 2005). An even more indirect and uncertain, but possibly more efficient approach, consists of systematic searches for directional and temporal coincidences of GRBs and SN from the cross correlation of GRBs and SN catalogs (Wang \& Wheeler 1998; Hudec et al. 1999). These searches are clearly affected by i) the large uncertainties in the GRB position (typically of a few degrees) (e.g. Kippen et al. 1998) leading to possible serious overestimates of the number of real associations; but also ii) the completeness and flux limit of the SN/GRB catalogs used. Nevertheless, if the indications by Zeh et al. (2004) prove to hold, catalog cross-correlations should indeed find evidence for a significant number of real associations.

In principle a more powerful method for selecting candidate associations from the catalog cross correlation could be envisaged if GRB associated with SN were characterized by particular temporal and/or spectral properties with respect to the whole of the GRB population. Indeed Norris et al. (1999) explored the hypothesis that there exists a subclass of GRBs related to SN whose $\gamma$-ray prompt light curve presents a single smooth peak, similar to that of GRB 980425 (as suggested by Bloom et al. 1998), but with negative results.

Here we focus on examining the possible peculiarities of the candidate events by investigating the temporal and spectral properties of GRBs for which there is an indication of being associated with a SN as a "class". More specifically we considered direct and indirect (tentative) associations, the latter ones based either on the light curve re-brightening or on the positional-temporal coincidence from catalog cross correlations. To this aim we searched the literature for the most up-to-date sample of GRB/SN candidates including the three robust cases of association (GRB 980425, GRB 030329, GRB 031203) and the 11 cases where the afterglow light curve re-brightening gives evidence for a coincident supernova explosion, that is GRB 021211 (Della Valle et al. 2003), GRB 011121 (Garnavich et al. 2003), GRB 020410 (Levan et al. 2005), GRB 041006 (Stanek et al. 2005) and seven GRBs examined by Zeh et al. (2004) (GRB 970228, GRB 980703, GRB 990712, GRB 991208, GRB 000911, GRB 010921, GRB 020405). From the available data we find that the light curve of these events consists of a single peak in $50 \%$ of cases and that their spectral parameters indicate a soft low energy spectrum. Given these suggestions we then extended the number of candidates by including those from both past catalogue cross-correlations and updating them using more recent GRB and SN catalogs.

Clearly, if GRBs in the sample turned out to be statistically (although not individually) characterized by any peculiar property - with respect to a random GRB sample - this would have interesting implications: i) it would indicate the existence of a SN-related sub-class of GRBs; ii) it would provide an empirical tool for efficiently identifying such events; iii) most importantly, it would offer clues on the physical understanding of their origin (indicating a mechanism different from that underlying GRB without SN signatures).

The outline of the paper is the following. The selected GRB sample is described in Sect. 2 and its general properties are discussed in Sect. 3. In Sects. 4 and 5 we present the detailed temporal and spectral analysis. We discuss in Sect. 6 the reliability of the GRB/SN associations and the nature of the selected GRB by means of (two of) the phenomenological intrinsic relations found for GRB with estimated redshift. Section 7 summarizes our conclusions. We adopt a $\Lambda$ CDM cosmology with $\Omega_{\Lambda}=0.7, \Omega_{\mathrm{m}}=0.3, H_{0}=65 \mathrm{~km} \mathrm{~s}^{-1}$.

\section{Sample selection}

We describe in detail the (heterogeneous) sample considered here, which includes the BATSE GRBs with indications of association with SN and for which temporal and spectral information could be retrieved, for a total of $36 \mathrm{GRB} / \mathrm{SN}$. The whole sample with its basic properties is listed in Table 1 and for simplicity hereafter referred to as the GRB/SN sample. The three robust associations based on the spectroscopic identification of a SN, namely GRB 980425, GRB 031203 and GRB 030329 will be considered as reference cases throughout this work.

The GRB/SN sample contains:

i) seven GRB/SN suggested by a bump in the late-time optical afterglow (Bloom et al. 1999; Zeh et al. 2004), robust spatial and temporal association (GRB 980425) or an indication of a line feature in X-rays (Piro et al. 1999), or exceptional SN properties (high luminosity, peculiar spectrum; e.g. Germany et al. 2000). Of all the claimed cases we included those with available BATSE data;

ii) $15 \mathrm{GRB} / \mathrm{SN}$ from the previous searches for temporal and spatial coincidence based on the cross-correlation of BATSE and SN catalogs: 11 pairs by Wang \& Wheeler (1998) and four pairs by Hudec et al. (1999);

iii) $14 \mathrm{GRB} / \mathrm{SN}$ identified in this work from an update of the catalog cross-correlation (including approximately the dates previously not covered, from Nov. 20, 1997 to May 26, 2000). In this period the BATSE GRB cata$\log ^{1}$ contains $721 \mathrm{GRB}$ and the SN catalog of IAUC ${ }^{2}$ reports $495 \mathrm{SN}$.

The catalog cross correlation method that we adopted is based on the following criteria. For the positional coincidence we required the SN to be within the BATSE GRB error box to which a constant 1.6 degrees of rms systematic error has been added (see e.g. Meegan et al. 1996). The exact SN phase at detection is not known (typically the optical maximum is reached in $\sim 20$ days) and furthermore no robust guess is possible for the delay between the SN and GRB explosions, as any estimate is strongly model dependent: a few months up to few

\footnotetext{
1 http://cossc.gsfc.nasa.gov/batse/BATSE_Ctlg/ index.html

2 http://cfa-www.harvard.edu/iau/lists/SN.html
} 
years are allowed in the supranova model (Vietri \& Stella 1998) while the explosions occur almost simultaneously in the collapsar model (e.g. MacFadyen \& Woosley 1998). Although the latter case is theoretically and observationally favored (e.g. Della Valle 2004, for a review), a large temporal window has been allowed for the possible correlation of the two events, requiring the $\mathrm{SN}$ date to be between -10 to +30 days with respect to the GRB trigger. No further constraints were imposed in the selection of either events and thus the results are biased by the SN sky distribution and the detection incompleteness at faint magnitudes; we considered both long and short GRB and no limit was imposed on the GRB count rate. Also, despite the indications from the three robust associations, we did not impose any selection on the SN type in our search (see however Sect. 5).

Note that in the previous analysis based on catalog cross correlations different search conditions were imposed. Wang \& Wheeler (1998) searched for SN with coordinates within $2 \sigma$ BATSE error boxes and supposed different time delays (according to the SN type) between SN and GRB. They also limited their search to $\mathrm{SN}$ at $z<0.1$. However, as this limit would exclude two (GRB 030329 and GRB 031203) out of the three more robust associations we did not impose any redshift cut. Hudec et al. (1999) reported the correlations for SN detected within the GRB error box up to 30 days after the GRB. Thus the compiled sample is not homogeneous as different criteria were applied. We considered re-examining the associations for the periods examined by Wang \& Wheeler (1998) and Hudec et al. (1999). However the BATSE instrumental trigger parameters have been changed during the BATSE 9 yr activity (Paciesas et al. 1999), until February 1997, when they were fixed. Therefore, a re-analysis would have been affected by this further bias, and thus not led to any increased completeness of the GRB/SN sample.

This updated cross-correlation yields 16 new GRB/SN coincident events. However, coordinates from the IPN ${ }^{3}$ for two GRB revealed that the associations were spurious (and thus excluded from the sample). In Fig. 1 error boxes and time delays are displayed for $31 \mathrm{GRB} / \mathrm{SN}$ (two GRBs had multiple associations and three GRBs had an indication of an underlying $\mathrm{SN}$ from the afterglow observations).

The significance of such blind (catalog based) searches was studied by Kippen et al. (1998), who assigned a significance to the observed correlation statistics by generating a set of random GRB catalogs. They find no evidence for association for GRBs having precise BATSE/Ulysses localizations, although they could not exclude it for a subset of weak bursts. While these results point toward a low chance to select real coincidences with such a procedure, the peculiarity of some properties of the GRB/SN sample (shown below) instead suggests that such a method does not select a random sub-sample of BATSE GRBs.

\footnotetext{
${ }^{3}$ http://www.ssl.berkeley.edu/ipn3/interpla.html
}

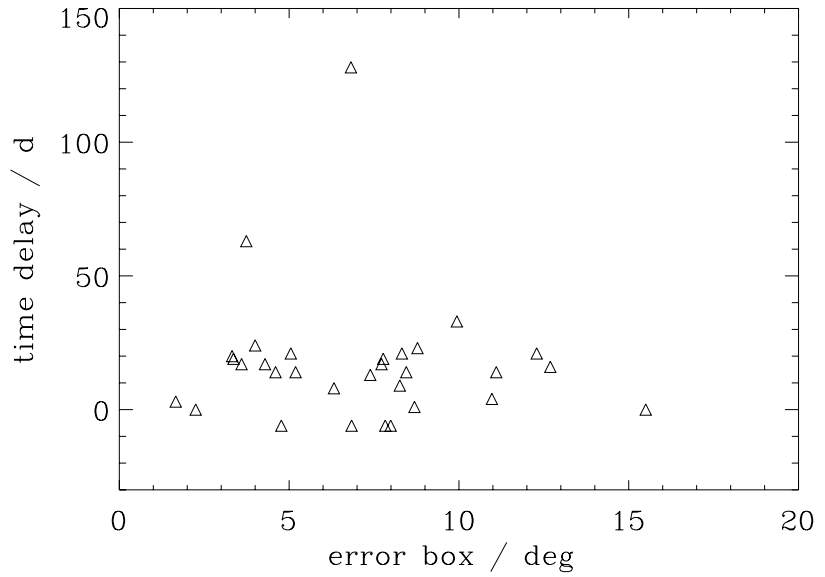

Fig. 1. BATSE error boxes and time delays for the GRB/SN sample. The time delay is between the SN discovery date and the GRB event.

\section{GRB/SN sample properties}

Based on the properties of GRB 980425 it has been suggested (see e.g. Bloom et al. 1998) that, if there is a physical connection between a sub-sample of GRBs and SN, such GRBs might exhibit distinct characteristics. Therefore we have compared the global properties of the GRB/SN sample with those of the entire BATSE sample.

No qualitative (nor statistical) differences are found as far as duration (represented by $T_{90}$ ), spectral hardness (HR32) ratio and total burst fluence are concerned (see Fig. 2, top and bottom panels), as both the range and shape of the GRB/SN distributions are indistinguishable from those of the rest of the GRBs in the BATSE catalog.

As expected from the typical worsening of the positional accuracy with decreasing photon statistics (see Kippen et al. 1998), the GRBs identified by the catalogs crosscorrelations are mostly weak, with typical peak (50-300 keV) flux $<1.5$ phot $\mathrm{cm}^{-2} \mathrm{~s}^{-1}$ (see Fig. 2).

While no peculiarity is found for the above quantities in the GRB/SN sample, in the next section we present a more detailed analysis of their GRB temporal profile and spectra ${ }^{4}$. Notice that the bias on the flux as well as the selection criteria of the GRB/SN sample result in "non optimal" statistics for the temporal and spectral analysis. This in turn requires a careful selection of a BATSE GRB "control sample", as previous analysis of BATSE GRBs has been typically performed on brighter samples.

\section{Light curve analysis}

In this section we present the analysis of the temporal profiles of the GRB/SN sample and compare the results with the (known) properties of larger GRB control samples.

While the temporal profiles of GRBs display a wide diversity (see e.g. Norris et al. 1999) we recall for future reference some of the important systematic findings on their properties: (1) flux peaks occur later at lower energies (spectral

\footnotetext{
${ }^{4}$ Note that in the following the GRB/SN events reported in each case/figure is different, depending on the quality of the available data.
} 

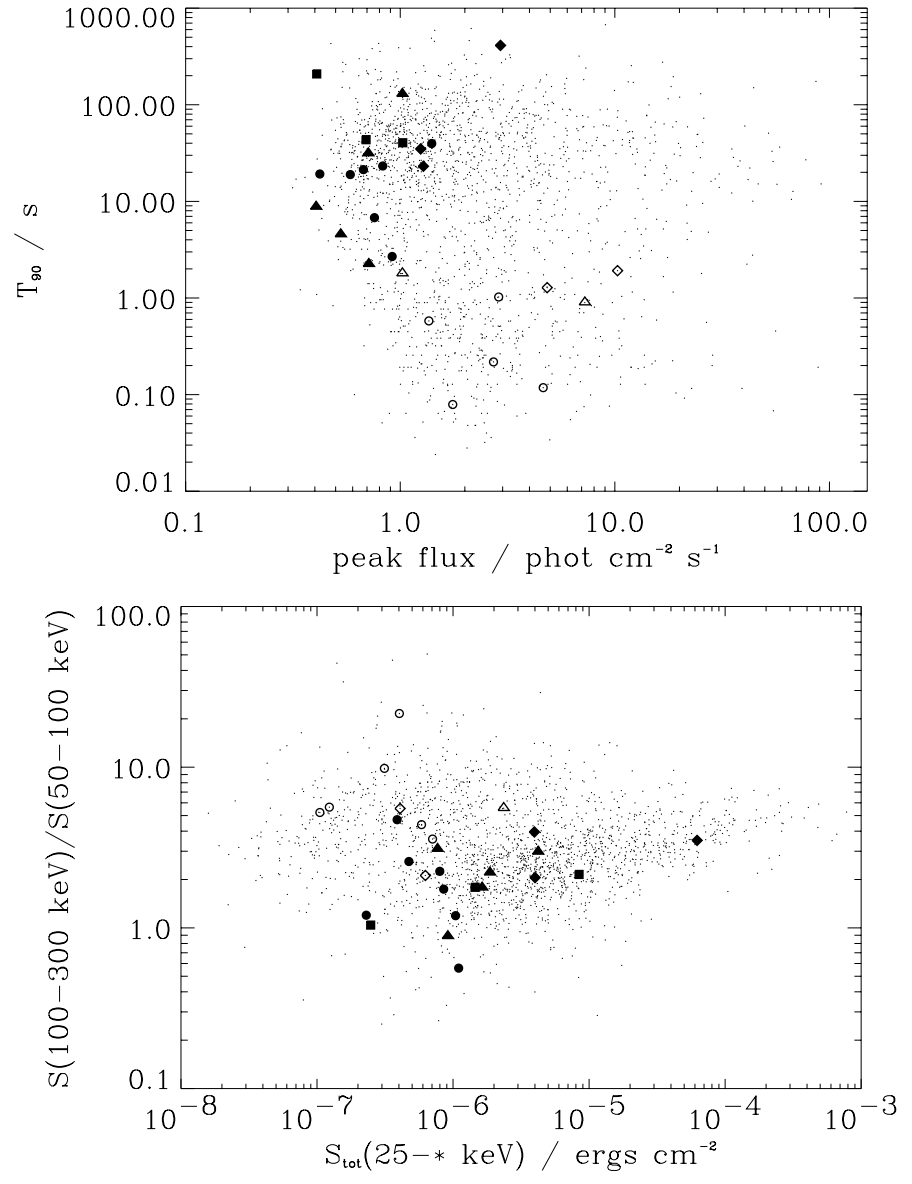

Fig. 2. Comparison of the durations $T_{90}$, peak fluxes, hardness ratios (i.e. HR32) and total fluences of the GRB/SN and the entire BATSE samples. Triangles, squares and circles indicate the tentative associations found by Wang \& Wheeler (1998), Hudec et al. (1999) and this work, respectively. Diamonds are the GRB with an indication of a $\mathrm{SN}$ event in the afterglow. Filled symbols represent long $\operatorname{GRB}\left(T_{90}>2 \mathrm{~s}\right)$.

lags - Norris et al. 1996; Norris et al. 2000); (2) the pulse parameter distributions are consistent with lognormal distributions (Quilligan et al. 2002); (3) less variable GRBs are found to be intrinsically less luminous (variability-luminosity correlation, Fenimore \& Ramirez-Ruiz 2000).

\subsection{Analysis}

For most of the GRBs (16/36) we analyzed the time profiles from the BATSE concatenated 64-ms data derived from the eight BATSE Large Area Detectors (LADs); these data represent the detector count rates in four energy channels (25-50, $50-100,100-300$ and $>300 \mathrm{keV})$. We fitted both the energyintegrated flux and the individual four energy channel light curves, according to the count statistics available for each event. The duration of six events was shorter than $2 \mathrm{~s}$ : in these cases we could thus analyze the Time-Tagged Event (TTE) data with 2 -ms time resolution (which are buffer-size limited ${ }^{5}$ )

\footnotetext{
${ }^{5}$ http://cossc.gsfc.nasa.gov/batse/batseburst/tte/ index.html
}

integrated over the four energy channels. In the analysis of these data we also accounted for the $\approx 30 \mathrm{~ms}$ offset between trigger time (as reported in the BATSE catalog) and TTE data load time. For the remaining $14 \mathrm{GRB} / \mathrm{SN}$ events either no reprocessed light curves were found in the public archive (nine cases) or the low statistics of the light curve did not allow any reasonable fit (five cases).

First we determined for each GRB the background count rate selecting two time intervals not containing the burst ${ }^{6}$, and modeled it with a 2 nd order polynomial. We then built a pulse model by adding the best fit background model to the lognormal pulse profile (Brock et al. 1994) and fit the light curve as:

$I(t)= \begin{cases}A_{\max } \exp \left[-\frac{1}{2}\left(\frac{\log \left(t-t_{0}\right)-\mu}{\sigma}\right)^{2}\right], & t>t_{0} \\ 0 & \text { otherwise }\end{cases}$

where $\mu$ and $\sigma$ are the mean and standard deviation, respectively, and the time is scaled to the pulse onset $t_{0}{ }^{7}$. Two reasons are behind this choice of profile: 1) most of the GRBs in the sample appear as single-peaked (on the $64 \mathrm{~ms}$ and $2 \mathrm{~ms}$ timescale for long and short bursts, respectively); 2) it allows a direct comparison with the results of previous studies (e.g. Norris et al. 1999). The best fits $\left(\chi^{2} \sim 0.83-1.18\right)$ are obtained for the integrated light curves, and in 11 (out of total 22) cases an acceptable fit was obtained for individual channel light curves.

\subsection{Results}

The resulting distribution of $\sigma$ is shown in Fig. 3 (top panel) for all the pulses in the light curves of the 22 GRBs for which the fit was performed. The distribution of $\sigma$ vs. $\mu$ is reported in the bottom panel. As expected short bursts are characterized by $\sigma$ lower than long ones and their pulses are less asymmetric. While the statistics are rather poor, we note a global tendency for an anti-correlation between $\sigma$ and $\mu$ both in long and short $\mathrm{GRB} / \mathrm{SN}$ : there is no pulse with large $\mu$ (i.e. long rise time) and large $\sigma$ (i.e. very asymmetric), namely they are typically FRED-like (i.e. Fast Rise Exponential Decay).

In order to compare the GRB/SN pulse properties with a control sample we also examined the distribution of their FWHM (64 ms time resolution). In Fig. 4 this is shown together with the distributions derived by Norris et al. (1999) and Quilligan et al. (2002). The latter, referring to the brightest (long) 319 BATSE GRBs, is very broad, peaking around $0.7 \mathrm{~s}$, and significantly different from that of the GRB/SN sample. Interestingly, however, the $F W H M$ distribution for the subset of long GRB/SN events (solid line in Fig. 4) appears to be similar to that derived by Norris et al. (1999) from the analysis of a sample of long, single-peaked GRB. Indeed a K-S test ${ }^{8}$

\footnotetext{
${ }^{6}$ http://cossc.gsfc.nasa.gov/batse/batseburst/ sixtyfour_ms/ bckgnd_fits.html

7 The lognormal pulse model is skewed to the right (as determined by $\sigma)$.

8 While formally the results by Norris et al. (1999) refer to fits of count rates from channels $\mathrm{CH} 2+3$, typically the signals in $\mathrm{CH} 1$ and $\mathrm{CH} 4$ are weaker, thus not significantly affecting the comparison.
} 

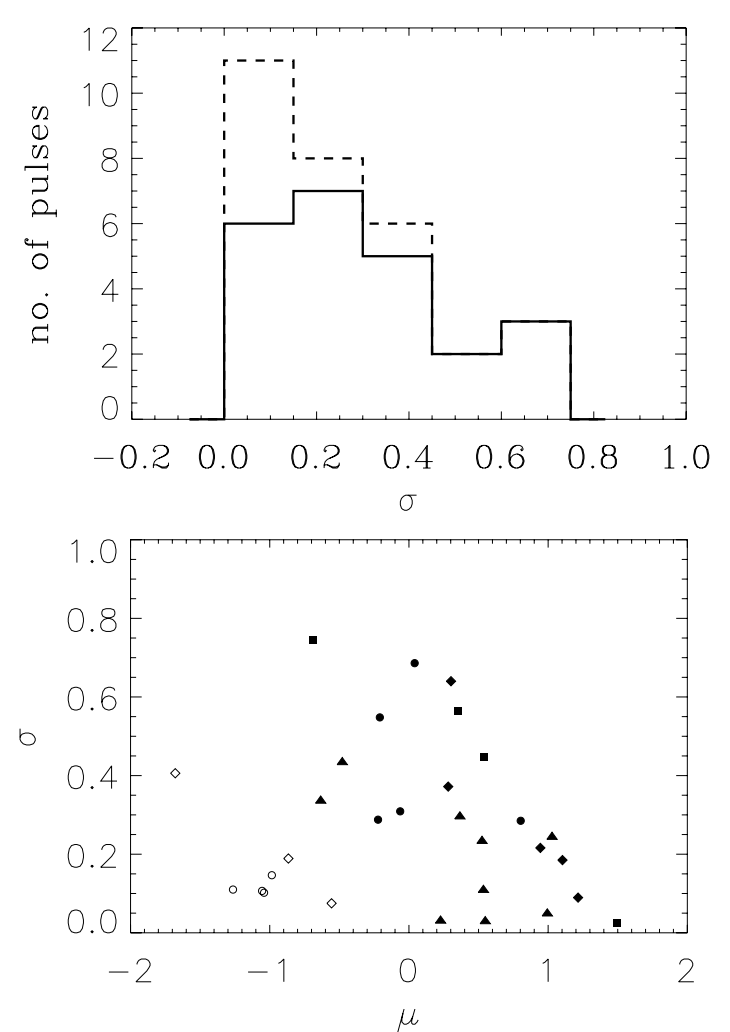

Fig. 3. Top: distribution of $\sigma$ for the lognormal pulse model. The solid and dashed lines represent the parameters for long bursts and the contribution of short bursts. All the pulses in the time history are represented. Bottom: $\sigma$ vs. $\mu$, i.e. the mean in log time. Symbols as in Fig. 2.

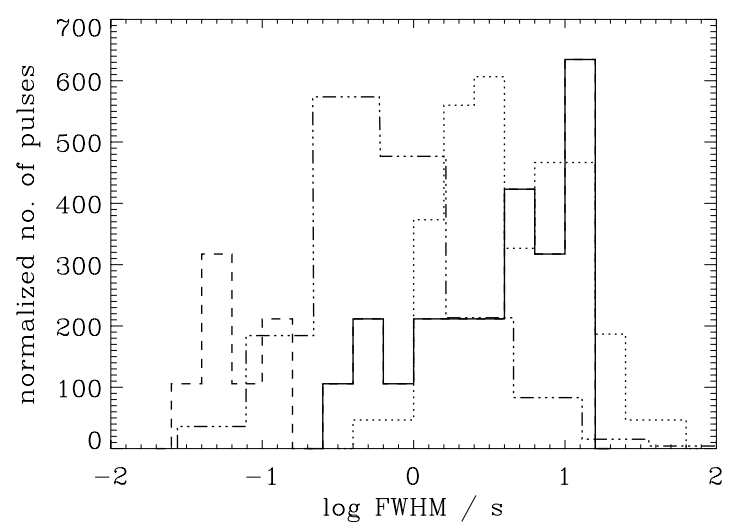

Fig. 4. Distributions of full widths at half maximum. The solid/dashed lines represent the contribution of long/short GRBs. The dotted line refers to the single-peaked bursts analyzed by Norris et al. (1999), while the dash-dotted line are the bright, long bursts analyzed by Quilligan et al. (2002). For clarity the distributions are normalized to the maximum number of data points (i.e. long bursts of Quilligan et al. 2002).

returns (for long, single-peaked GRB/SN) a probability $P_{\mathrm{KS}}=0.98$ that the two data sets originate from the same parent distribution.

In Fig. 5 (top panel) the pulse amplitudes (only for a $64 \mathrm{~ms}$ resolution timescale) vs. FWHM are displayed. Quilligan et al. (2002) find a weak negative correlation between these parameters. While there is no evidence for a trend for the whole GRB/SN sample, an anti-correlation (Spearman rank
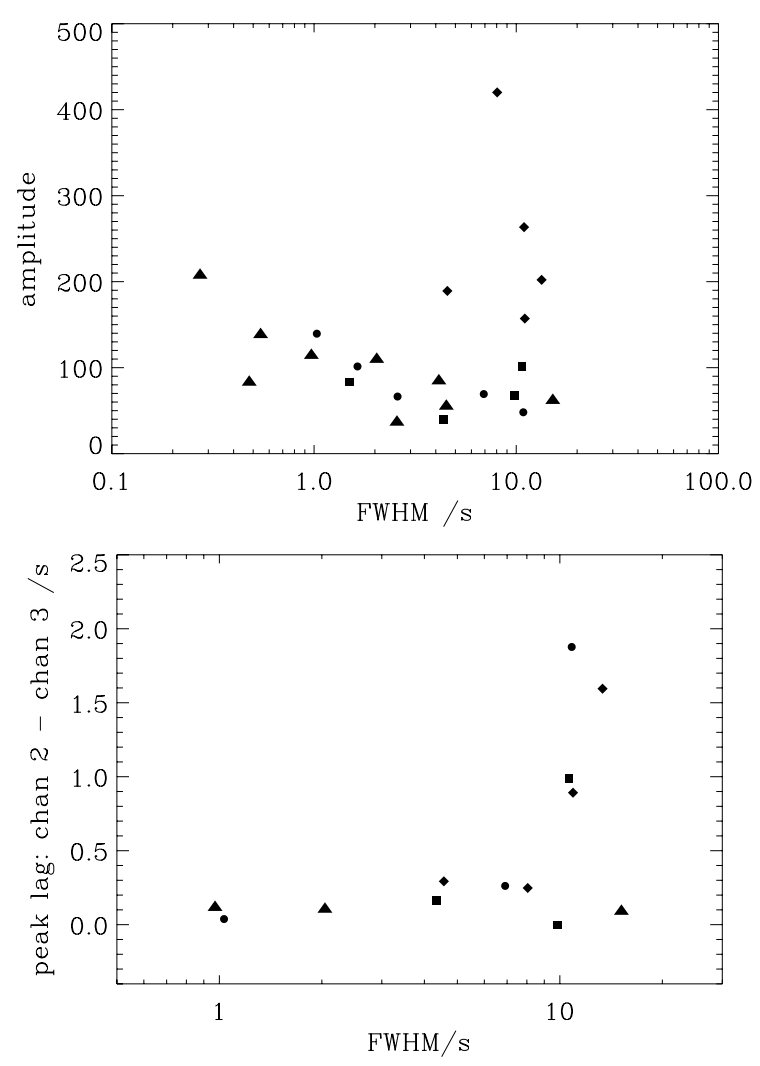

Fig. 5. Top: amplitude (fitted at $64 \mathrm{~ms}$ resolution) vs. FWHM as obtained from the fit to the total counts in the four channels. Bottom: Time lag between fitted peaks (in $\mathrm{CH} 2$ and $\mathrm{CH} 3$ ) vs. FWHM. Only long bursts are shown. Symbols as in Fig. 2.

correlation coefficient of -0.65 and probability 0.003 ) is present among the GRB/SN selected on the basis of the crosscorrelation only. The interpretation of this is not clear: it might be simply due to the bias toward low fluxes in GRB/SN selected from the catalog cross-correlation and/or indicate that indeed the latter are largely spurious associations, not representing (atypical) properties of GRB physically associated with $\mathrm{SN}$ events.

A tendency for wider pulses to have larger time lags appears instead for the GRB/SN sample (Fig. 5, bottom panel). There the spectral lag (i.e. the delay between the peaks of the low with respect to the high energy channel) is plotted against the FWHM of the fitted pulse. This behavior is consistent with what is reported by Norris et al. (1996) on the basis of a sample of 46 bright, long bursts: they found that the lag results mostly from the shift in the pulse centroid and thus the correlation is induced by the narrowing of the pulses toward higher energies. Different interpretations have been proposed for such a property (e.g. Salmonson 2000; Schaefer 2004) and very recently it has been suggested that it might be accounted for in the jet-viewing-angle scenario (Ioka \& Nakamura 2001) where both (small) pulse amplitudes and (long) spectral lags are related to (large) viewing angles of the jet. As shown in the top and bottom panels of Fig. 5, in the case of the GRB/SN sample (but excluding the seven GRB/SN determined by the light curve bumps) small amplitude pulses could correspond to large spectral lags. 
Thus, within the limited statistics and differences in the selection criteria of the GRB/SN sample, the GRB/SN events appear to be broadly similar to the whole of the BATSE GRB population. However, the analysis of the FWHM distributions highlighted one intriguing possibility, namely that the GRB/SN events behave more typically like single-peaked GRBs. Although aware that the bias toward weak GRB in the sample might be responsible for the above finding - Norris et al. (1999) cautioned that dim bursts might have a multiple pulse structure that is difficult to detect - we explored this aspect in detail.

\subsection{A connection between single-peaked GRBs and SN?}

As already mentioned, Bloom et al. (1998) suggested that GRBs associated with SN might have distinctive temporal and spectral properties, similar to those of GRB 980425, namely a smooth, single peaked, long-duration $\left(T_{90}=34.8 \mathrm{~s}\right)$ light curve and faint emission above $300 \mathrm{keV}$. Norris et al. (1999) considered this issue and searched the BATSE catalog with these criteria, but no other event similar to GRB 980425 and spatially/temporally correlated with any known $\mathrm{SN}$ was found. In our GRB/SN sample we instead found that the large majority $(\sim 80 \%)$ of GRB are single peaked. Of those not selected by the catalog cross-correlation, five out of seven GRBs are single-peaked events. Of the other two spectral associations, GRB 031203 shows a single peaked light curve (Soderberg et al. 2004), while GRB 030329 presents different temporal properties, i.e. a profile with two peaks of similar intensity (Vanderspek et al. 2004).

To quantitatively test whether the GRB/SN sample comprises an unusually high number of single-peaked GRBs (1) it has been necessary to determine an objective way of characterizing the "single-peakness" and (2) due to the limited size of the GRB/SN sample (29 GRBs with $64 \mathrm{~ms}$ data) the probability of finding a similar large percentage of single-peaked events had to be computed via random extractions of GRB sub-samples from the BATSE catalog.

\subsection{Method}

We thus built an algorithm that identifies single-peaked bursts in the BATSE catalog. As the time profiles of GRBs differ to a great extent it is difficult to have a unique approach applicable to every burst: eventually we imposed conditions to determine whether the burst has one or more than one peak.

Li \& Fenimore (1996) proposed a method for identifying peaks in GRB time histories, and a modification of this was also applied by Nakar \& Piran (2002) in the temporal analysis of short bursts. It consists of defining a count bin $C_{\mathrm{p}}$ at time $t_{\mathrm{p}}$ as a candidate peak if it satisfied the condition:

$C_{\mathrm{p}}-C_{1,2} \geq N_{\text {var }} \sqrt{C_{\mathrm{p}}}$,

where $C_{1,2}$ are neighboring count bins at times $t_{1,2} . N_{\text {var }}$ determines how rigorous the search is: too large $N_{\text {var }}$ might clearly result in an algorithm insensitive to faint peaks; too low $N_{\text {var }}$ could identify statistical variations as peaks. Previous works showed that the choice of $N_{\text {var }}=5$ optimizes the algorithm.

The search was performed on the background-subtracted light curves, and thus a linear background was fitted to data before and after the burst trigger, and subtracted. The resulting time histories were binned to $128 \mathrm{~ms}$ resolution.

As one of the problems encountered was that sharp structures in the time history could be mis-identified as peaks, we applied a wavelet analysis to determine the dominant modes of variability. A wavelet transform is convenient to use when the analyzed signal consists of short spikes that are not well approximated when doing a Fourier analysis: the wavelet coefficients resulting from the convolution of the signal with the wavelet basis functions represent the signal variation at a particular resolution (see e.g. Kolaczyk 1997; Quilligan et al. 2002 , for details). We reconstructed the original signal by keeping only the maxima of the wavelet transforms; a very limited number ( 25$)$ of the wavelet coefficients was used to reproduce the signal. Although this does not guarantee the generation of a smooth light curve, with this approach the unique peak finding condition could be applied for the whole set of GRBs.

The search for peaks was limited to the burst region, excluding periods before the burst that may contain a precursor. We tested several choices for peak finding, optimizing it to recognize what would be visually identified as a single-peaked GRB, and implemented the slightly modified peak-finding conditions: a) adopting the value $N_{\text {var }}=7$; and b) adding the condition for a peak to have at least $20 \%$ more flux than the neighboring "valleys".

\subsection{Results}

We applied the algorithm to the GRB/SN sample and this defined 23 (out of 29) single-peak bursts: this differs by less than $10 \%$ from the number we find by visual inspection.

To establish the chance probability of such occurrence, we selected GRBs from the BATSE catalog having fluences in the range spanned by the GRB/SN events (between $6.9 \times 10^{-8}$ and $8.4 \times 10^{-6} \mathrm{erg} \mathrm{cm}^{-2}$ ), ending up with $1587 \mathrm{GRBs}$, and systematically applied the algorithm to their 64-ms resolution data (for 142 out of them the algorithm did not identify any peak, as a consequence of the rigorous conditions imposed). We then randomly extracted sub-samples of the same size $(\mathcal{N}=29)$ of the GRB/SN sample, repeating the extraction $n$ times. In Fig. 6 we show the distribution of the fraction of single-peak GRBs for $n=50,150$ and 250 .

We fitted a lognormal function to the probability distribution $^{9}$, i.e.:

$P(x)=\frac{1}{\sqrt{2 \pi} \sigma x} \mathrm{e}^{\left(-(\ln (x)-\mu)^{2} / 2 \sigma^{2}\right)}$,

for the case $n=250$ and find a $0.4 \%$ probability of having 23/29 single-peaked bursts. If the number of single-peaked bursts in the sample were $10 \%$ lower (i.e. 20 or more events)

\footnotetext{
9 Lognormal distributions have been used to describe the distributions of various GRB properties (see e.g. Li \& Fenimore 1996; Quilligan et al. 2002).
} 


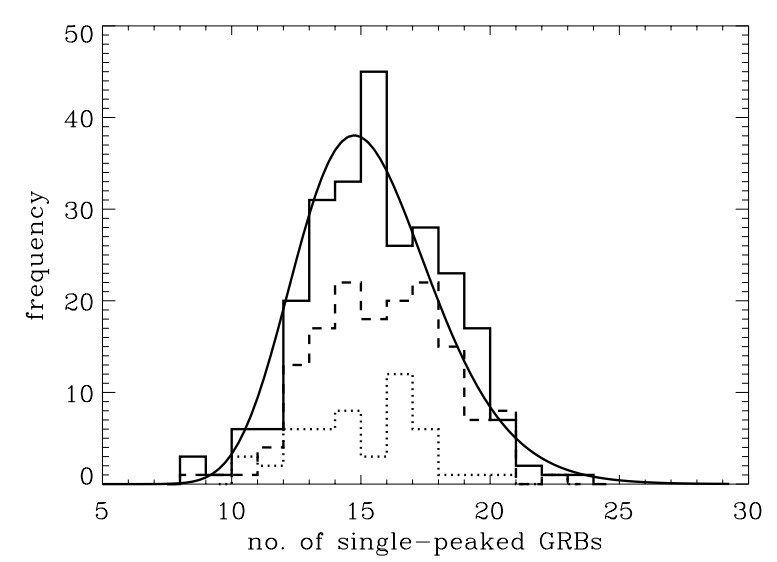

Fig. 6. Distributions of the number of single-peaked GRB in subsamples of $\mathcal{N}=29$ GRB. The dotted, dashed and solid histograms represent the distributions for respectively $n=50,150,250$ extractions out of the total sample of 1445 bursts. The lognormal fit corresponds to the case $n=250$.

the probability of observing it would still be $\sim 5 \%$. Note that the distribution has a maximum for $\sim 15$ single-peaked bursts; this is consistent with the result by Norris et al. (1999) who found $68 / 116$ bursts with at least one pulse - suggesting however that it is difficult to assign an exact number of pulses as they observed a continuum of lower emissions superposed on otherwise single-peaked events.

The homogeneous treatment of the GRB/SN and random control sub-samples should ensure that the above result is unbiased against effects that may vary the number of detected pulses in the GRB time history, such as the rigorous conditions on peak candidates, the fluence range, the (still present) spikes mis-interpreted as pulses and the presence of short GRBs which in fact appear to be multi-peaked when seen at $2 \mathrm{~ms}$ resolution (there were $23 \%$ short bursts in the sample).

\section{Spectral analysis}

Let us now consider whether GRB/SN show any peculiarity in terms of spectral properties. We recall that the spectrum of GRB 980425 is "unusual", peaking at $\sim 100 \mathrm{keV}$ (Jimenez et al. 2001), below the typical $300 \mathrm{keV}$ peak energy. Also GRB 030329 is characterized by a similar low peak energy (Vanderspeck et al. 2004), while GRB 031203 has a higher peak energy (>212 keV, Sazonov et al. 2004; see however Watson et al. 2005).

GRB spectra in the BATSE energy range (25-1800 keV) are usually described via the phenomenological Band representation (Band et al. 1993), which consists of two smoothly connected low- and high-energy power laws. Despite of a nonnegligible dispersion of the fitted parameters, the typical values for the low and high energy power-laws photon indices are $\alpha \sim-1$ and $\beta \sim-2.5$, respectively, and the spectral peak energy $E_{\text {peak }}$ (i.e. the peak in a $v F_{\nu}$ representation) is around a few hundred keV (Preece et al. 2000). The Band model in general successfully represents both the time integrated as well as the time resolved spectra (Ford et al. 1995).
Following the widely adopted methodology described by Preece et al. (2000), we analyzed the GRB/SN spectra obtained by integrating the signal over the burst duration (a time resolved spectral analysis was possible only in a few cases due to the low signal-to-noise ratio).

\subsection{Analysis}

The spectral analysis was heavily limited because in many cases the GRB signal was too low for any reasonable spectral fit. For just over half of the bursts (16) we used the High Energy Resolution Burst (HERB) data from the most brightly illuminated BATSE LAD (128 energy channels). For short bursts or in those cases with no good time coverage with the HERB data we analyzed the Medium Energy Resolution (MER) data (five cases), which consist of 16-energy channel spectra with a time resolution of $16 \mathrm{~ms}$ during the first half of the total duration and $64 \mathrm{~ms}$ during the second half.

The background spectrum was extracted and averaged from two time intervals, before and after the burst trigger. The time interval for the spectrum accumulation typically starts at the trigger and lasts for the duration as estimated by $T_{90}$. The spectrum was eventually re-binned in energy in order to have enough statistics to apply the $\chi^{2}$ goodness-of-fit test ${ }^{10}$.

The spectra were analyzed using the XSPEC v.11.1.0 package, performing the fitting with the Band model in the $\sim 30-\sim 1700 \mathrm{keV}$ energy range. In some GRBs this model fit resulted in unconstrained spectral parameters and/or unacceptable reduced $\chi^{2}$ (18 cases): if mainly due to the low statistics at high energies we tested as alternative models the cutoffpower law (CPL; for one GRB) and broken power-law (BPL; six GRBs) spectral functions, while if due to a low signal in most channels, we adopted the simpler single power-law model fit (11 GRBs). The same set of models was applied to larger samples of bright bursts (Preece et al. 2000): we stress that our aim is to characterize the spectra of the GRB/SN sample rather than establish which model preferentially fits them (in fact the choice of a particular model may not be unique even for bright bursts, e.g. Ghirlanda et al. 2002). We considered the model fit acceptable when a reasonable $\chi^{2}$ (ranging from 0.8 to 1.3 ) and non systematic residuals were obtained. All the models (except for the single power-law) are characterized by a low energy power-law $\propto E^{\alpha}$, a high energy power-law (Band and BPL models) $\propto E^{\beta}$ and a characteristic energy $E_{\text {peak }}$.

\subsection{Results}

The distributions of the spectral parameters are shown in Fig. 7 for the GRB/SN and the Preece et al. (2000) sample ${ }^{11}$.

\footnotetext{
${ }^{10}$ We binned in order to obtain a minimum of 5 counts/bin. This (minimal) choice has been tested by re-binning the data for a minimum of 10 and 20 counts/bin (e.g. Bevington \& Robinson 1992) and the corresponding fits did not yield either significantly different $\chi^{2}$ or significantly different fit parameters.

${ }^{11}$ For the comparison the results of the time-resolved spectral analysis by Preece et al. were averaged, as for most GRB/SN it was possible to analyze only time integrated spectra.
} 

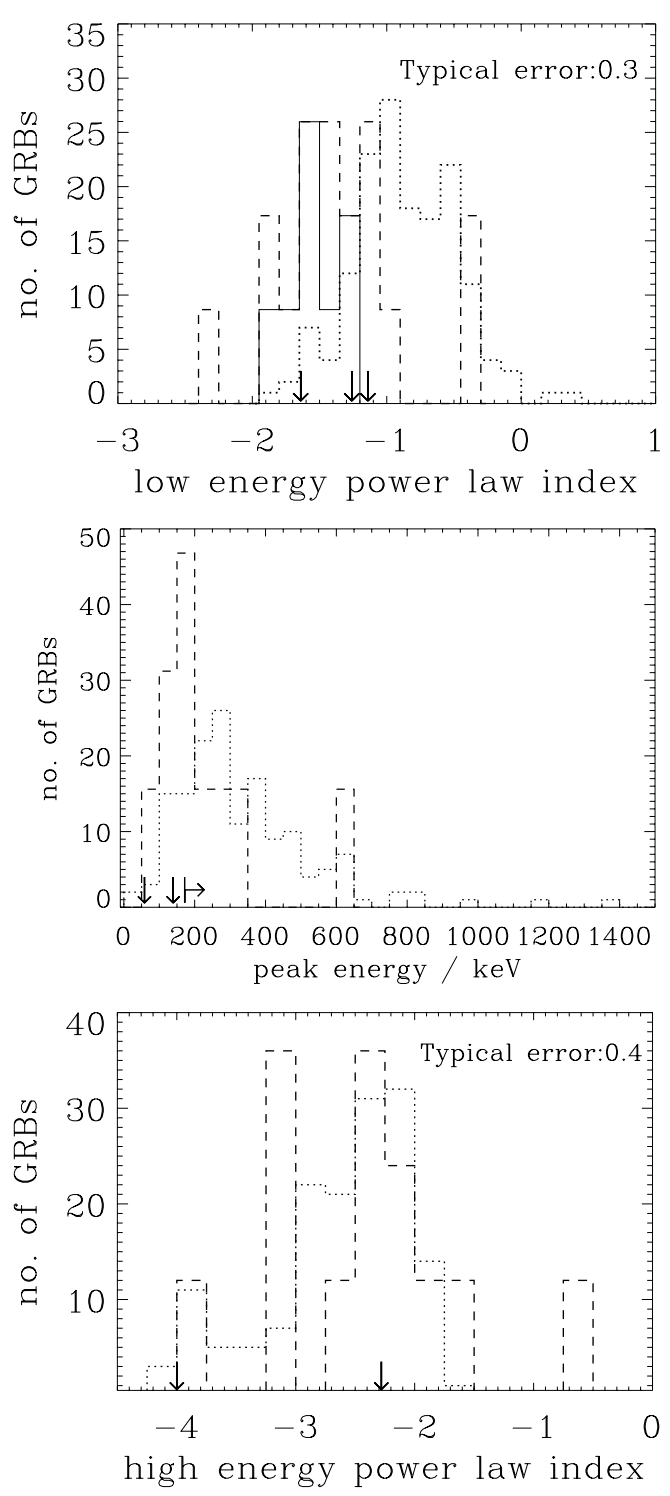

Fig. 7. Spectral parameter distributions obtained for the GRB/SN (dashed lines) and GRBs examined by Preece et al. (2000) (dotted lines). For clarity the number of GRB/SN events is normalized to the total number of GRBs in the larger control sample by Preece et al. (2000). In the top panel the results obtained by fitting a single power law model are indicated separately (solid line). A high energy powerlaw index $\beta=-4$ refers to the exponential function fit. Errors for the peak energy range from 20 to $\sim$ a few hundred $\mathrm{keV}$. The arrows in each panel indicate the values (or limits) corresponding to GRB 980425, GRB 030329 and GRB 031203. Note that the highest values in the three distributions refer to GRB 990810 and GRB 971221, found by catalog cross-correlation.

For the GRB/SN sample the average spectral index is $\alpha \sim$ -1.5 , with almost no bursts with $-1<\alpha<0$, which are instead found in the comparison sample. This suggests that on average GRB/SN events have low energy spectra softer than typical GRBs. Note that GRB 980425 and GRB 030329 have spectra fitted with $\alpha \sim-1.2$ (Vanderspek et al. 2004) and GRB 031203 is modeled with a single power law with $\alpha \sim-1.6$ (Sazonov et al. 2004). The single power law model (fitted to $\sim$ half of GRBs) in general gives a value of $\alpha$ lower than that inferred from "convex" models (Band, CPL or BPL). A K-S test performed on the $\alpha$ values obtained by fitting the "convex" models only yields that the GRB/SN sample is significantly different from that reported by Preece et al. (2000), with $P_{\mathrm{KS}}=0.03$.

The distributions of $E_{\text {peak }}$ (for the ten GRB/SN events that had a spectrum fitted by Band, CPL or BPL) are reported in the middle panel of Fig. 7. The typical peak energy for GRB/SN is $\sim 220 \mathrm{keV}$, lower than that of bright BATSE GRBs (from Preece et al. 2000) with $P_{\mathrm{KS}}=0.04$. As mentioned, two out of three GRBs with spectroscopic evidence of association lie in the lowest bin of this distribution (see Fig. 7).

Finally we report in Fig. 7 (bottom panel) the distributions of $\beta$. Within the large errors ( $\beta$ is usually not well constrained because of the $\mathrm{S} / \mathrm{N}$ decrease in the high energy channels), there is no evidence of deviations with respect to bright long bursts.

\section{Redshift estimates}

Clearly a crucial test of the physical connection of our GRB/SN pairs would be the determination of the GRB redshifts. To date, redshifts are known only for $\sim 42$ GRBs via the optical emission lines of their host galaxies or absorption lines in the optical transient spectra. Due to the high $\gamma$-ray flux, unaffected by extinction, and to the limited number of afterglow detections, several attempts have been made to find a reliable method for deriving distances from the prompt $\gamma$-ray emission only. Unfortunately the large diversity in the temporal and spectral properties of GRBs and the related uncertainties result in significant uncertainties in any such method. For our purposes however even an indication of the GRBs distance could rule out or reinforce an association.

Several correlations have been proposed to infer the GRB energetics on the basis of observable temporal and spectral $\gamma$-ray properties. Fenimore \& Ramirez-Ruiz (2000; see also Reichart et al. 2001) found a correlation between the luminosity and a variability indicator for a few GRBs with measured $z$ : smoother bursts appear to be intrinsically less luminous. Another distance indicator was identified by Norris et al. (2000) as a correlation between GRB luminosities and spectral lags. These correlations were used to estimate redshifts for a large sample of GRBs (Fenimore \& Ramirez-Ruiz 2000). Another interesting correlation has been found by Amati et al. (2002; see also Lloyd-Ronning \& Ramirez-Ruiz 2002) between the intrinsic peak energy $E_{\text {peak }}$ and the isotropic total radiated energy $E_{\mathrm{rad}}$. Alternative methods to determine $z$ have been proposed: Atteia (2003) identified a method by combining the spectral parameters and GRB duration; Bagoly et al. (2003) suggested a correlation between the peak flux ratios and $z$.

The calibration of all these relations is based on GRBs with known $z$, but always excluding GRB 980425 due to its peculiar energetics and temporal/spectral properties. Also GRB 031203 turned out to be outside of some of those correlations. Caution should be thus applied in using the above results for candidate GRB/SN events, which might indeed be most similar to GRB 980425 and GRB 031203.

In the following we focus on two such methods for which we could infer more reliably the corresponding GRB parameters, namely the Lag-Luminosity correlation 
(Norris et al. 2000) and the Peak Energy-Energy correlation (Amati et al. 2002).

\subsection{Lag-Luminosity correlation}

Norris et al. (2000) found that the spectral time lag $\tau$ is anti-correlated with the isotropic peak luminosity $L_{\text {iso }}$, namely $L_{\text {iso }, 53} \approx 1.3 \times(\tau / 0.01 \mathrm{~s})^{-1.15}$ for a sample of six bursts with known $z$. In order to derive the lag measure for the GRB/SN the same methodology was applied. In particular: i) we calculated the cross correlation functions (CCFs) of the time histories in two different energy channels (see also Band 1997), i.e. CH1 and $\mathrm{CH} 3$, adding the apodization intervals before and after the burst (apodization intervals had a constant count number, computed as average value in adjacent time bins); ii) the analysis was limited to light curve regions with count rates higher than $10 \%$ of the peak luminosity; iii) the time series were binned in order to have higher $\mathrm{S} / \mathrm{N}$ (64 ms data were binned to $128 \mathrm{~ms}$; for short GRBs TTE data were binned to $4 \mathrm{~ms}$ ); iv) we fitted a linear background to each channel separately in the non-burst portions of the data and subtracted it from the total counts; v) the CCFs were fitted with a third order polynomial around their peaks in order to derive an estimate of the time lag, then corrected by ${ }^{12}$ a factor $1 /(1+z)$. We derived the peak luminosity in the $50-300 \mathrm{keV}$ energy range directly by integration of the GRB fitted spectrum for each possible redshift.

The results are presented in Fig. 8 (top panel). Unfortunately it was possible to estimate time lags for only three bursts (16 GRB/SN have both spectral data and fourchannels time histories available and of those most have negligible signal in $\mathrm{CH} 1$; also in three cases the obtained time lags were negative and thus not further considered). The curves represent the intrinsic (i.e. corrected for $z$ ) $L_{\text {iso }}$ and $\tau$ assuming a range $z=0.01-4$ for the possible redshift. The points on them indicate the values of $L_{\text {iso }}-\tau$ corresponding to the redshift of the putative associated $\mathrm{SN}$, when known. It appears that the GRB/SN events could be consistent with the Lag-Luminosity correlation only if they were placed at high $z(>1.5)$ indicating that the associations are spurious. Alternatively, these GRBs might not obey such a correlation and thus could still be located at the SN redshift. The latter (intriguing) possibility is supported by the fact that these GRBs would then mimic the characteristics of two out of the three spectroscopic GRB/SN (i.e. GRB 980425 and GRB 031203), possibly forming a separate class.

\subsection{Peak Energy-Energy correlation}

The Peak Energy-Isotropic Energy correlation (Amati et al. 2002) has the form $E_{\text {peak }} \propto E_{\text {rad }}^{0.52}$. Similarly to the procedure described above we computed for the GRB/SN the total bolometric fluence (see Amati et al. 2002) by integrating $\left(1-10^{4} \mathrm{keV}\right.$ ) the best fit spectrum (11 GRB/SN events). The results are presented in the $E_{\text {peak }}-E_{\text {rad }}$ plane in Fig. 8 (bottom panel). The position of the symbols again indicates the corresponding values

\footnotetext{
12 Note that both time dilation and channel energies should be corrected for $z$ (see e.g. Fenimore \& Ramirez-Ruiz 2000).
}
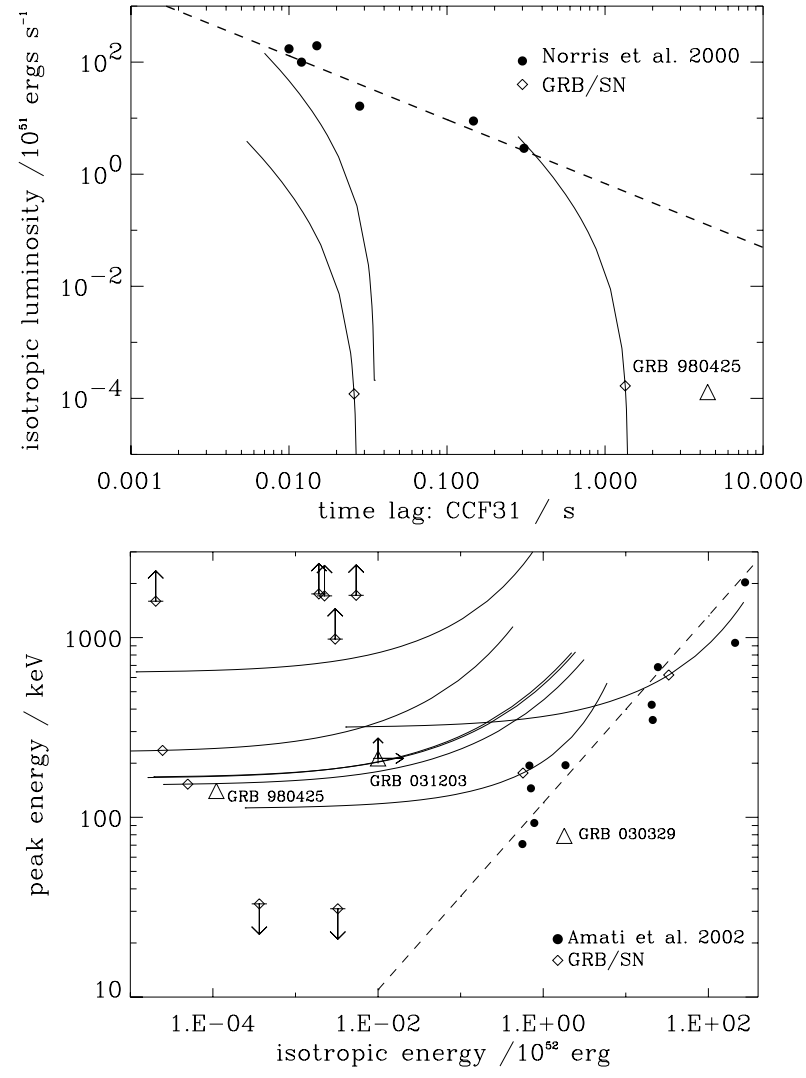

Fig. 8. Top panel: relation by Norris et al. (2000). Bottom panel: relation by Amati et al. (2002). In both panels, the GRB/SN events are marked (diamonds) at the values corresponding to the redshift of the associated SN, while the trajectories represent the values corresponding to different redshifts, in the range 0.01-4. The dashed lines indicate the correlations as determined by Norris et al. (2000) and Amati et al. (2002). GRB/SN events which had only upper or lower limits on their peak energy are shown only for the values corresponding to the redshift of the coincident $\mathrm{SN}$ as arrows.

for the SN redshift, while the curved lines show the values for the interval $z=0.01-4$. The GRB/SN events for which only lower/upper limits of the peak energy could be estimated are shown only at the $z$ of the possibly associated SN. We also mark the values for the three spectroscopic associations. Only two of the GRB/SN could be consistent with the Amati et al.'s relation: GRB 980703 (at $z=0.967$, Djorgovski et al. 1998), and GRB 971221 which might be associated with a SN at $z \approx 0.58$ (see Table 1). The properties of the rest of GRB/SN are not consistent with those predicted by such a relation, but most importantly in ten cases (five of which with well determined parameters) could not obey the correlation (within its scatter) for any z. As known, GRB 980425 and GRB 031203 are also clear outliers of the Amati relation (whereas GRB 030329 is consistent with it), i.e. of the strong associations, $2 / 3$ do not follow it (with respect to about 40 GRBs with known redshift).

Within the limitations given by the (small) fraction of the GRB/SN sample we find that these events do not follow the Lag-Luminosity and the Peak Energy-Isotropic Energy correlations, in some cases for any assumed $z$. This - supported by the similarity to the behavior of GRB 980425 and GRB 031203 
- appears to indicate that at least a few GRB/SN events form a separate class with respect to the majority of long GRBs.

\section{Summary and conclusions}

We presented a detailed analysis of the temporal and spectral properties of a sample of BATSE GRBs with an indication to be associated with a SN event, with the aim of determining whether these reveal any peculiar property with respect to the bulk of the detected GRBs.

The "GRB/SN sample" was obtained combining GRB associations based on the presence of re-brightening in the afterglow light curves which might be interpreted as SN signatures and (more tentatively) on positional/temporal coincidence from the cross correlation of GRB and SN catalogs. The latter ensemble was expanded with respect to previous works by including the most recent and complete GRB and SN catalogs. The whole set comprises 36 GRBs (three of which lack a corresponding SN). For GRBs with public data available we performed a systematic analysis and compared their temporal and spectral properties with those of larger, "control" samples of BATSE bursts.

The analysis of the temporal profiles revealed that in most cases the light curve presents a single peak. This property has been considered by different authors (also on theoretical grounds) as a possible distinctive feature of GRBs associated with SN events (Bloom et al. 1998; Norris et al. 1999). The chance probability of finding the same fraction of single peaked bursts from a random extraction from the BATSE archive is $\sim 0.4 \%$.

We further analyzed the time profiles fitting a lognormal model and correlated the pulse parameters. Dim bursts turned out to have time lags larger than bright ones, as possibly explained by a larger viewing angle (Ioka \& Nakamura 2001). This interpretation has also been invoked in the case of GRB 980425/SN 1998bw and GRB 031203/SN 2003lw to account for their low luminosity compared to the bulk of GRBs (Waxman 2004).

In terms of the spectral properties the GRB/SN sample shows softer spectra (both in terms of low energy spectral index and peak energy) with respect to typical bursts. This might be a distinctive feature of GRBs associated with SN as two out of three spectroscopic associations also share this atypical low peak energy.

Finally, in an attempt to estimate their redshift, we considered two of the intrinsic correlations proposed for the population of long bursts, namely the Lag-Luminosity (Norris et al. 2000 ) and the $E_{\text {peak }}-E_{\text {rad }}$ (Amati et al. 2002) relations. While some GRB/SN might be consistent with these if not associated with the putative $\mathrm{SN}$, some events do not obey such correlations for any redshift. This might indicate that they are effectively different or that these correlations only hold for particular subsamples of GRBs (e.g. bright long bursts - see also Ghirlanda et al. 2004, who find that short bursts mostly populate a region above the $E_{\text {peak }}-E_{\text {rad }}$ correlation). In particular, the properties of this handful of GRB/SN again closely resemble those of two out of three spectroscopic associations which are also outliers of those relations.
As discussed in the Introduction, previous results as well as the large positional uncertainties and wide time window for the selection of $\mathrm{GRB} / \mathrm{SN}$ via catalog cross-correlation in fact point toward a low probability of finding true associations via this method. However, the above results are suggestive of the opposite. Statistically, the GRB/SN sample contains (with respect to the whole of the BASTE events) an unusually high fraction of single peaked bursts, with softer spectra and some of those do not follow two of the proposed correlations for GRBs. Most of these properties mimick those of the spectroscopic GRB/SN associations. Although it is not obvious how to classify individual GRB/SN in order to see any intersection among GRB/SN having "unusual" characteristics, in Table 1 we schematically listed the three "unusual" properties for each GRB/SN (single-peakness, spectrum and consistency with the $E_{\text {peak }}-E_{\text {rad }}$ relation). In 19 GRBs (other than GRB 980425) there are at least two properties that set the event as GRB 980425-like: ten GRB/SN have two or three (three of those are short and only one not selected by the catalog crosscorrelation); six GRBs have two but not the third one (five are short and two not selected by the catalog cross-correlation).

A final indication that the catalog cross-correlations are not randomly sampling GRB and $\mathrm{SN}$ events comes from the properties of the selected $\mathrm{SNe}$. We did not restrict our search for correlations on the basis of the SN type, yielding a significant number of SNe Ia in the sample (see Table 1). While indeed it has been also proposed on theoretical grounds that type Ia SN might also be associated with bursts (e.g. Berezinsky et al. 1996; Dar \& De Rujula 2004), the current view (theoretically and observationally based) is that (some long) GRBs are likely associated with type Ibc SNe. An intriguing finding is that among the GRB/SN events found by the catalog crosscorrelations, type Ib,c SNe comprise about $28 \%$ of the events (i.e. 8/29), which is about four times larger than expected from SN catalog statistics (see Valenti et al. 2005, who examined the Asiago SN catalog). This result also confirms the analysis independently carried out by Valenti et al. (2005) on the BATSE catalog; indeed, these authors found an excess of Ib,c types among SNe possibly associated with BATSE GRBs $(\sim 17 \%$, with a significance level of $\sim 93 \%$ ).

While the uncertainty on the reality of a single association remains high, these results indicate that: 1) there is a population significantly larger than that detected so far of GRB 980425-like events associated with SN; 2) the corresponding GRB have "atypical" temporal and spectral properties (and thus may suggest that not all GRBs are associated with at least typical "SN"); 3) they appear under-luminous in their observed $\gamma$-ray emission (at the level of $0.1-1 \%$ ) with respect to "standard" GRBs.

All three aspects give statistical and physical information on the nature of GRB 980425-like events. The finding of a significant number of these under-luminous nearby bursts (e.g. viewed off axis) might indicate from statistical arguments that these comprise the majority of GRBs (e.g. Sazonov et al. 2004). From a physical point of view, in one promising scenario accounting for a qualitatively softer spectrum, a smooth, singlepeak light curve and an apparently under-energetic event could be envisaged if the line of sight is at large angles with respect 
Table 1. The GRB/SN sample. The line separates GRBs with BATSE error boxes smaller and larger than 8 deg. $\left({ }^{*}\right)$ Refers to a single-peaked GRB with substructure. There is no SN reported when there was only a SN bump present in the afterglow or in case of multiple (\#) associations. ${ }^{\circledR}$ IAUC SN catalog. ${ }^{+}$Already in the Amati et al. (2002) sample; ${ }^{1}$ Wang \& Wheeler (1998); ${ }^{2}$ Hudec et al. (1999); ${ }^{3}$ Piro et al. (1999); ${ }^{4}$ Germany et al. (2000); ${ }^{5}$ Bloom et al. (1999); ${ }^{6}$ Galama et al. (1998); ${ }^{7}$ Holland et al. (2001); ${ }^{8}$ Rigon et al. (2003); ${ }^{9}$ Terlevich et al. (1999).

\begin{tabular}{|c|c|c|c|c|c|c|c|c|c|c|c|c|c|c|c|c|c|}
\hline GRB & RA & Dec & $\begin{array}{c}\text { Error } \\
\text { box (deg) }\end{array}$ & $\begin{array}{c}T_{90} \\
\mathrm{~s} \\
\end{array}$ & $\begin{array}{c}P_{64 \mathrm{~ms}} \\
\mathrm{ph} / \mathrm{cm}^{2} \mathrm{~s}\end{array}$ & $\begin{array}{c}F_{>25 \mathrm{keV}} \\
10^{-6} \mathrm{erg} / \mathrm{cm}^{2}\end{array}$ & $\begin{array}{l}\text { Single } \\
\text { peak* }^{*}\end{array}$ & $\alpha$ & SN & RA & Dec & $\begin{array}{c}\text { Discovery } \\
\text { date }\end{array}$ & Type & $z^{@}$ & $\begin{array}{c}\text { Follow } \\
E_{\text {peak }}-E_{\mathrm{rad}} \\
\end{array}$ & $\begin{array}{c}\text { Catalog } \\
\text { cross-corr. }\end{array}$ & Ref. \\
\hline 920321 & 184.5 & 5.7 & 3.6 & - & - & - & $\mathrm{N}$ & $-1.06_{-0.13}^{+0.14}$ & 1992Q & 182.0 & -1.6 & 920407 & - & - & $\mathrm{N}$ & $\bar{Y}$ & 2 \\
\hline 920613 & 312.9 & -55.6 & 4.6 & 129.0 & 1.02 & 1.86 & $\mathrm{~N}$ & - & 1992ae & 322.1 & -61.6 & 920627 & Ia & 0.075 & $\mathrm{~N}$ & $\mathrm{Y}$ & 1 \\
\hline 920708 & 308.3 & -49.9 & 3.3 & 3.2 & - & - & $\mathrm{Y}^{*}$ & $-1.41_{-0.17}^{+1.54}$ & 1992al & 311.5 & -51.4 & 920727 & Ia & 0.014 & $\mathrm{~N}$ & $\mathrm{Y}$ & 1 \\
\hline 920628 & 317.8 & -27.3 & 7.7 & 4.5 & 0.52 & 1.63 & $\mathrm{Y}$ & - & 1992at & 321.8 & -37.0 & 920717 & Ia & - & - & $\mathrm{Y}$ & 1 \\
\hline 920925 & 129.7 & -58.7 & 5.0 & - & - & - & $\mathrm{Y}$ & - & $1992 b g$ & 115.5 & -62.5 & 921016 & Ia & 0.036 & - & $\mathrm{Y}$ & 1 \\
\hline 951107 & 148.8 & 39.8 & 4.0 & 43.5 & 0.69 & 1.45 & $\mathrm{Y}^{*}$ & $-1.62_{-0.10}^{-0.11}$ & $1995 b c$ & 147.7 & 40.3 & 951201 & II & 0.048 & $\mathrm{~N}$ & $\mathrm{Y}$ & 2 \\
\hline 960221 & 47.8 & -31.2 & 4.3 & 31.3 & 0.71 & 4.22 & $\mathrm{Y}$ & - & $1996 \mathrm{~N}$ & 54.7 & -26.3 & 960310 & $\mathrm{Ib}$ & - & - & $\mathrm{Y}$ & 1 \\
\hline 961029 & 59.3 & -52.6 & 3.3 & 40.4 & 1.03 & 8.44 & $\mathrm{Y}$ & - & 1996bx & 59.7 & -53.3 & 961118 & Ia & 0.069 & - & $\mathrm{Y}$ & 2 \\
\hline 970508 & 132.4 & 80.6 & 2.3 & 23.1 & 1.28 & 3.96 & $\mathrm{Y}$ & $-1.46_{-0.23}^{+0.10}$ & - & - & - & - & - & 0.835 & $\mathrm{Y}^{+}$ & $\mathrm{N}$ & 3 \\
\hline 970514 & 67.6 & -60.9 & 3.7 & 1.3 & 4.83 & 0.41 & $\mathrm{Y}$ & - & 1997cy & 68.0 & -61.7 & 970716 & II & 0.0642 & - & $\mathrm{N}$ & 4 \\
\hline 971218 & 116.1 & 16.7 & 5.2 & 6.8 & 0.76 & 1.09 & $\mathrm{Y}$ & $-1.25_{-0.58}^{+0.31}$ & 1998B & 116.5 & 18.7 & 980101 & Ia & 0.045 & $\mathrm{~N}$ & $\mathrm{Y}$ & \\
\hline 971221 & 73.7 & 4.7 & 6.3 & 1.0 & 2.87 & 0.58 & $\mathrm{Y}$ & $-2.35_{-1.56}^{+0.57}$ & 1997ey & 74.2 & -2.6 & 971229 & Ia & 0.58 & $\mathrm{Y}$ & $\mathrm{Y}$ & \\
\hline 980326 & 133.3 & -18.6 & 2.1 & - & - & - & $\mathrm{Y}^{*}$ & $-1.90_{-0.20}^{+0.26}$ & - & - & - & - & - & $0.9-1.1$ & $\mathrm{Y}^{+}$ & $\mathrm{N}$ & 5 \\
\hline 980425 & 291.9 & -53.1 & 1.6 & 34.8 & 1.24 & 4.0 & $\mathrm{Y}$ & $-1.14_{-0.21}^{+0.22}$ & 1998bw & 293.7 & -52.8 & 980428 & Ic & 0.0085 & $\mathrm{~N}$ & $\mathrm{~N}$ & 6 \\
\hline 980525 & 157.6 & -17.8 & 6.8 & 39.6 & 1.40 & 0.38 & $\mathrm{~N}$ & $-1.69_{-0.19}^{+0.18}$ & 1998ce & 152.6 & -25.8 & 980519 & II & - & - & $\mathrm{Y}$ & \\
\hline 980703 & 359.1 & 12.0 & 0.5 & 411.6 & 2.93 & 62.2 & $\mathrm{~N}$ & $-1.01_{-0.12}^{+0.10}$ & - & - & - & - & - & 0.967 & $\mathrm{Y}$ & $\mathrm{N}$ & 7 \\
\hline 980910 & 195.1 & -21.1 & 6.8 & - & - & - & - & - & $1999 \mathrm{E}$ & 199.3 & -18.5 & 990115 & IIn & 0.025 & - & $\mathrm{N}$ & 8 \\
\hline 990527 & 199.9 & 49.3 & 7.7 & 18.9 & 0.58 & 1.04 & $\mathrm{Y}$ & $-1.65_{-0.40}^{+0.33}$ & $1999 \mathrm{ct}$ & 198.3 & 46.2 & 990613 & Ia & 0.18 & - & $\mathrm{Y}$ & \\
\hline 990810 & 358.1 & 1.7 & 4.3 & 0.1 & 4.63 & 0.70 & $\mathrm{Y}$ & $-0.32_{-0.40}^{+0.40}$ & & multiple & (2) & associations & & & $\mathrm{N}$ & $\mathrm{Y}$ & \\
\hline 991002 & 25.1 & 3.7 & 2.2 & 1.9 & 10.2 & 0.62 & $\mathrm{Y}$ & $-1.12_{-0.26}^{+0.49}$ & $1999 \mathrm{eb}$ & 25.7 & 3.7 & 991002 & IIn & - & $\mathrm{N}$ & $\mathrm{N}$ & 9 \\
\hline 991015 & 11.6 & 8.9 & 7.8 & 2.7 & 0.91 & 0.47 & $\mathrm{Y}$ & - & $1999 \mathrm{ef}$ & 14.7 & 12.7 & 991009 & Ia & - & - & $\mathrm{Y}$ & \\
\hline 991123 & 157.2 & 26.9 & 4.7 & - & 2.95 & 0.31 & $\mathrm{Y}$ & - & $1999 \mathrm{gj}$ & 10.5 & 26.1 & 991117 & Ia & 0.018 & - & $\mathrm{Y}$ & \\
\hline 000114 & 121.6 & 39.5 & 7.9 & 0.58 & 1.35 & 0.12 & $\mathrm{Y}$ & $-1.42_{-0.21}^{+0.21}$ & $2000 \mathrm{C}$ & 114.2 & 35.2 & 000108 & Ic & 0.012 & $\mathrm{~N}$ & $\mathrm{Y}$ & \\
\hline 000415 & 199.4 & -29.9 & 7.4 & 0.22 & 2.72 & 0.40 & $\mathrm{Y}$ & $-0.39_{-0.49}^{+0.74}$ & $2000 \mathrm{ca}$ & 203.8 & -34.1 & 000428 & Ia & 0.024 & $\mathrm{~N}$ & $\mathrm{Y}$ & \\
\hline 910423 & 196.7 & -5.2 & 11.1 & 208.5 & 0.41 & 0.25 & $\mathrm{Y}$ & $-1.88_{-0.51}^{+0.40}$ & 1991aa & 190.5 & -6.0 & 910507 & $\mathrm{Ib}$ & - & - & $\mathrm{Y}$ & 1 \\
\hline 950917 & 339.9 & -1.47 & 8.2 & - & - & - & $\mathrm{Y}$ & - & $1995 \mathrm{ac}$ & 341.4 & -8.8 & 950926 & Ia & 0.05 & - & $\mathrm{Y}$ & 2 \\
\hline 960925 & 29.3 & -13.9 & 8.4 & 1.8 & 1.02 & 0.47 & $\mathrm{Y}$ & - & 1996at & 17.1 & -1.0 & 961009 & $\mathrm{Ib} / \mathrm{c}$ & 0.09 & - & $\mathrm{Y}$ & 2 \\
\hline 961218 & 97.7 & -21.7 & 12.7 & 8.7 & 0.40 & 0.91 & $\mathrm{Y}^{*}$ & - & 1997B & 88.3 & -17.9 & 970104 & Ic & 0.01 & $\mathrm{~N}$ & $\mathrm{Y}$ & 2 \\
\hline 970907 & 346.9 & 11.2 & 8.3 & 0.9 & 7.24 & 2.35 & $\mathrm{Y}$ & $-1.26_{-0.16}^{+0.14}$ & $1997 \mathrm{dg}$ & 355.1 & 26.2 & 970928 & Ia & 0.03 & $\mathrm{~N}$ & $\mathrm{Y}$ & 2 \\
\hline 971013 & 167.0 & 2.7 & 8.8 & 12.3 & - & - & $\mathrm{Y}$ & - & $1997 d q$ & 175.2 & 11.5 & 971105 & $\mathrm{Ib}$ & 0.003 & - & $\mathrm{Y}$ & 2 \\
\hline 971120 & 155.7 & 76.4 & 9.9 & 2.2 & 0.71 & 0.77 & $\mathrm{Y}$ & - & 1997ei & 178.5 & 58.5 & 971223 & Ic & 0.011 & - & $\mathrm{Y}$ & 2 \\
\hline 980530 & 148.3 & -27.3 & 8.7 & 21.3 & 0.67 & 0.79 & $\mathrm{Y}$ & $-1.51_{-0.15}^{+0.14}$ & $1998 \mathrm{ck}$ & 145.2 & -29.1 & 980531 & Ia & 0.038 & $\mathrm{~N}$ & $\mathrm{Y}$ & \\
\hline 990719 & 224.5 & 10.8 & 10.9 & 23.2 & 0.82 & 0.23 & $\mathrm{~N}$ & - & $1999 \mathrm{dg}$ & 227.8 & 13.5 & 990723 & Ia & - & - & $\mathrm{Y}$ & \\
\hline 990902 & 58.2 & 62.5 & 15.5 & 19.2 & 0.42 & 0.85 & $\mathrm{Y}$ & - & 1999dp & 67.3 & 69.5 & 990902 & II & 0.016 & - & $\mathrm{Y}$ & \\
\hline 991021 & 346.3 & -30.9 & 12.3 & - & 1.30 & 0.07 & $\mathrm{Y}$ & - & $1999 \mathrm{ex}$ & 334.0 & -36.8 & 991109 & Ic & 0.011 & - & $\mathrm{Y}$ & \\
\hline 000319 & 172.7 & -13.8 & 9.8 & 0.08 & 1.75 & 0.10 & Y & - & & multiple & (5) & associations & & - & - & $\mathrm{Y}$ & \\
\hline
\end{tabular}

to the jet axis and the observed GRB emission is in fact prompt emission reprocessed (reflected) at such angles by circumburst material. A detailed study of the spectral and temporal properties expected in such cases is ongoing.

Estimates of the bolometric energetics of GRBs (e.g. Soderberg 2004) and the improved statistics which will be allowed in the HETE, INTEGRAL and the upcoming Swift era offer the most obvious possibilities to test these results.

Acknowledgements. We thank the referee, E. Cappellaro, for useful criticisms and valuable suggestions. Z.B. thanks T. Maccarone for helpful comments. E.P. thanks P. Mazzali and M. Vietri for the initial discussions on this project. The Italian MIUR and INAF are acknowledged for financial support by Z.B., A.C. and G.G. (MIUR/COFIN Grant 2003020775-002). This research was supported in part by the National Science Foundation under Grant No. PHY99-07949; the KITP (Santa Barbara) is thanked for kind hospitality (AC). The research has made use of data obtained through the High Energy Astrophysics Science Archive Research Center online service, provided by the NASA/GODDARD Space Flight Center.

\section{References}

Amati, L., Frontera, F., Tavani, M., et al. 2002, A\&A, 390, 81 Atteia, J. L. 2003, A\&A, 407, L1

Bagoly, Z., Csabai, I., Mészáros, A., et al. 2003, A\&A, 398, 919 Band, D., Matteson, J., Ford, L., et al. 1993, ApJ, 413, 281

Band, D. L. 1997, ApJ, 486, 928

Berezinsky, V. S., Blasi, P., \& Hnatyk, B. I. 1996, ApJ, 469, 311

Bevington, P. R., \& Robinson, D. K. 1992, Data reduction and error analysis for the physical science, 2nd Ed. (McGraw-Hill)

Bloom, J., Kulkarni, S. R., Harrison, F., et al. 1998, ApJ, 506, L105 
Bloom, J., Kulkarni, S. R., Djorgovski, S. G., et al. 1999, Nature, 401, 453

Brock, M., Meegan, C., Wilson, R., et al. 1994, in Gamma-Ray Bursts, ed. G. J. Fishman, J. J. Brainerd, \& K. Hurlsy (New York: AIP), AIP Conf. Proc., 307, 672

Dar, A., \& De Rujula, A. 2004, Phys. Rep., 405, 203

Della Valle, M., Malesani, D., Benetti, S., et al. 2003, A\&A, 406, L33

Della Valle, M. 2004, in Gamma-Ray Bursts in the Afterglow Era, Conf. Proc., 4th Rome Workshop, in press

Djorgovski, S. G., Kulkarni, S. R., Bloom, J. S., et al. 1998, ApJ, 508, L17

Fenimore, E. E., \& Ramirez-Ruiz, E. 2000 [arXiv:astro-ph/0004176]

Ford, L. A. 1995, ApJ, 439, 307

Fynbo J. P. U., Sollerman, J., Hjorth, J., et al. 2004, ApJ, 609, 962

Galama, T., et al. 1998, Nature, 396, 670

Garnavich, P. M., Stanek, K. Z., Wyrzykowski, L., et al. 2003, ApJ, 582,924

Germany, L., Reiss, D. J., Sadler, E. M., Schmidt, B. P., \& Stubbs, C. W. 2000, ApJ, 533, 320

Ghirlanda, G., Celotti, A., \& Ghisellini, G. 2002, A\&A, 393, 409

Ghirlanda, G., Ghisellini, G., \& Celotti, A. 2004, A\&A, 422, L55

Hjorth, J., Sollerman, J., Møller, P., et al. 2003, Nature, 423, 847

Holland, S., Fynbo, J. P. U., Hjorth, J., et al. 2001, A\&A, 371, 52

Hudec, R., Hudcová, V., \& Hroch, F. 1999, A\&AS, 138, 475

Ioka, K., \& Nakamura, T. 2001, ApJ, 554, L163

Jimenez, R., Band, D., \& Piran, T. 2001, ApJ, 561, 171

Kippen, R. M., Briggs, M. S., Kommers, J. M., et al. 1998, ApJ, 506, L27

Kolaczyk, E. D. 1997, ApJ, 483, 340

Levan, A., Nugent, P., Fruchter, A., et al. 2005, ApJ, 624, 880

Li, H., \& Fenimore, E. 1996, ApJ, 469, L115

Lloyd-Ronning, N. M., \& Ramirez-Ruiz, E. 2002, ApJ, 576, 101

MacFadyen, A. I., \& Woosley, S. E. 1999, ApJ, 524, 262

Malesani, D., Tagliaferri, G., Chincarini, G., et al. 2004, ApJ, 609, L5

Meegan, C. A., Pendleton, G. N., Briggs, M. S., et al. 1996, ApJS, 106,65
Nakar, E., \& Piran, T. 2002, MNRAS, 330, 920

Norris, J., Nemiroff, R. J., Bonnell, J. T., et al. 1996, ApJ, 459, 393

Norris, J., Bonnell, J. T., \& Watanabe, K. 1999, ApJ, 518, 901

Norris, J., Marani, G. F., \& Bonnell, J. T. 2000, ApJ, 534, 248

Paciesas, W. S., Meegan, C. A., Pendleton, G. N., et al. 1999, ApJS, 122,465

Piro, L., Costa, E., Feroci, M., et al. 1999, A\&AS, 138, 431

Podsiadlowski, Ph., Mazzali, P. A., Nomoto, K., Lazzati, D., \& Cappellaro, E. 2004, ApJ, 607, L17

Preece, R. D., Briggs, M. S., Mallozzi, R. S., et al. 2000, ApJS, 126 , 19

Quilligan, F., McBreen, B., Hanlon, L., et al. 2002, A\&A, 385, 377

Reichart, D., Lamb, D. Q., Fenimore, E. E., et al. 2001, ApJ, 552, 57

Rigon, L., Turatto, M., Benetti, S., et al. 2003, MNRAS, 340, 191

Salmonson, J. D. 2000, ApJ, 544, L115

Sazonov, S. Yu., Lutovinov, A. A., \& Sunyaev, R. A. 2004, Nature, 430, 646

Schaefer, B. E. 2004, ApJ, 602, 306

Soderberg, A. M., Kulkarni, S. R., Berger, E., et al. 2004, Nature, 430, 648

Soderberg, A. M., Kulkarni, S. R., Fox, D. B., et al. 2005, ApJ, 627, 877

Stanek, K. Z., Matheson, T., Garnavich, P. M., et al. 2003, ApJ, 591, L17

Stanek, K. Z., Garnavich, P. M., Nutzman, P. A., et al. 2005, ApJ, 626, L5

Terlevich, R., Fabian, A., \& Turatto, M. 1999, IAUC, 7269

Valenti, S., Cappellaro, E., Della Valle, M., et al. 2005, Proc 4th Workshop Gamma Ray Bursts in the Afterglow Era, 28, 633

Vanderspek, R., Sakamoto, T., Barraud, C., et al. 2004, ApJ, 617, 1251

Vietri, M., \& Stella, L. 1998, ApJ, 507, L45

Wang, L., \& Wheeler, J. C. 1998, ApJ, 504, L87

Watson, D., et al. 2005, ApJ, in press

Waxman, E. 2004, ApJ, 602, 886

Woosley, S. E., Eastman, R. G., \& Schmidt, B. P. 1999, ApJ, 516, 788

Zeh, A., Klose, S., \& Hartmann, D. H. 2004, ApJ, 609, 952 